\title{
Analizando y seleccionando juegos del mundo para la educación científica y matemática
}

\author{
Carlos Sergio Gutiérrez Perera, Alicia Fernández Oliveras y María Luisa Oliveras. \\ Universidad de Granada \\ Recepción: 12 de abril de 2015 | Revisión: 30 de mayo de 2015 | Aceptación/Publicación: 24 de julio de 2015 \\ Correspondencia: oliveras@ugr.es | alilia@ugr.es | sergiogutierrezperera@gmail.com |http://hdl.handle.net/10481/37111
}

\begin{abstract}
Resumen: A través del presente trabajo pretendemos mostrar la utilidad del juego considerado como un método y un recurso para la enseñanza-aprendizaje de las ciencias y las matemáticas. El proceso de selección que ha dado lugar al catálogo general de juegos que mostramos se ha llevado a cabo de manera experimental, jugando desde una perspectiva científica y matemática para descubrir el potencial que estos juegos del mundo encierran. Hemos analizado juegos de diversas culturas, algunos de los cuales son ancestrales y se encuentran en peligro de desaparición, como parte de tradiciones culturales que son dignas de ser preservadas. Otros son originarios de grupos actuales que han desarrollado juegos. Con el trabajo resumido en este artículo hemos apreciado que la práctica de estos juegos es beneficiosa para los sujetos, que mejoran su actitud mostrándose más optimistas y felices jugando.
\end{abstract}

Palabras clave: Didáctica de las ciencias experimentales | Didáctica de las matemáticas | Juegos del mundo | Etnomatemáticas

\section{ANALYSING AND SELECTING WORLD GAMES FOR SCIENTIFIC AND MATHEMATICAL EDUCATION}

\begin{abstract}
Through this work we intend to show the utility of play considered as a method and a resource for teaching and learning science and mathematics. The game selection process that has led to the generic catalogue of games that we are presenting has been done experimentally, playing from a scientific and mathematical perspective to discover the potential that these cultural games contain. We have analysed ancestral games of diverse cultures that are about to disappear and have a cultural tradition worthy of being preserved. With the work summarised in this article we have seen that the practice of these games is beneficial to the subjects, whose attitude improves-becoming more optimistic and happier when playing.
\end{abstract}

Keywords: Science Education | Mathematical Education | World Games | Ethnomathematics

\section{Introducción}

La problemática abordada en el trabajo que resumimos en este artículo queda concretada en encontrar una manera de trabajar las matemáticas en el aula de forma que el alumnado descubra "otras matemáticas" de las que pueda apropiarse, logre aprendizajes significativos y una mejora sustancial de las competencias matemáticas, al tiempo que damos una respuesta adecuada a la realidad multicultural de las aulas y abogamos por la protección y difusión de juegos de mesa tradicionales con contenido matemático.

Muchos juegos de tablero del mundo constituyen en esencia muestras culturales de actividad matemática: prácticas etnomatemáticas en toda regla (Albizu, FernándezOliveras y Oliveras, 2014). Algunos de ellos han desaparecido, mientras que otros corren peligro de caer en el olvido. Se trata de un legado inmaterial que debemos proteger.

Por ello, presentamos en este texto un catálogo de juegos que hemos seleccionado pensando que pueden servir como recurso y método en un entorno educativo, tanto de ámbito formal como no formal, en el que acometer los objetivos de:

- que el alumnado descubra "otras matemáticas", y las practique; 
- que el alumnado desarrolle instrumentos mentales propios de la investigación y de la metodología de las ciencias experimentales;

- dar respuesta adecuada a la realidad multicultural de la sociedad y de las aulas como microescenario social.

Consideramos que toda educación en un contexto multicultural debe ser intercultural, pues pensamos que no es suficiente el respeto a la diversidad, sino que se debe favorecer el encuentro y la comunicación entre personas de diferentes culturas, tal como expresa Gómez-Lara (2003). Y creemos que el juego es un buen vehículo para la interculturalidad en la educación, a todos los niveles.

Entendemos el juego en el sentido de actividad que las personas realizan como necesidad que emana de la propia naturaleza racional y que a su vez les convierte en personas, de acuerdo con varios autores especializados (Decroly y Monchamp, 1983; Elkonin, 1985), entre los cuales consideramos la teoría de Huizinga como la más completa (Huizinga, 1972) y fundamento para nuestra propuesta de catálogo. Dicho catálogo facilita dar a conocer juegos que benefician a jóvenes y mayores, ejercitando un ocio inteligente y desarrollando una amplia variedad de habilidades, así como hacer que los juegos perduren como patrimonio de la humanidad.

\section{Desarrollo de la propuesta}

El juego es una actividad universal que desarrolla las capacidades científicas y humanas ya que implica reglas, estructuraciones lógicas, sanciones y recompensas, mientras que permite la negociación y el intercambio de puntos de vista entre las personas que juegan. Por todo ello nos hemos involucrado en la búsqueda de juegos que tengan estas características:

- Ser legado etnomatemático-científico propio de alguna cultura.

- Tener un buen potencial formativo en el campo de las ciencias y/o las matemáticas.

Nuestra propuesta se sitúa también en la línea de prevenir las dificultades que presenta la enseñanza-aprendizaje de las matemáticas y de las ciencias experimentales en el alumnado, tanto más cuando consideramos dichos procesos en cursos superiores.

La multifuncionalidad del juego, que se plasma en funciones como la exploratoria e iniciática, la simbólica, la socializadora e integradora, o la entretenedora, entre otras, se concreta en el juego formal en cuatro funciones básicas o razones para jugar:

- conocer,

- aprender,

- desarrollarse y

- divertirse.

Resulta claro que jugar puede entenderse como una forma de educarse, y que enseñar a jugar puede considerarse, por tanto, una manera de educar. Y educar en un modelo intercultural requiere preparación del profesorado y sobre todo recursos adecuados (Oliveras, 2006), por lo que estos juegos pueden ser de utilidad para el profesorado que desee realizar la educación científica desde un modelo intercultural (Fernández-Oliveras y Oliveras, 2014a, 2014b, 2015; Bishop, 1998). 
Mostraremos aquí el catálogo que hemos elaborado, y cuyo estudio desde el punto de vista educativo y curricular estamos iniciando, ya que consideramos que el lector puede beneficiarse del hecho de saber que los juegos incluidos son aptos para el logro de los objetivos descritos anteriormente, aunque no proporcionemos, todavía, un estudio detallado de las habilidades que pueden desarrollarse con los mismos.

Hemos experimentado el uso de estos juegos en entornos no formales, incluyendo una feria del conocimiento, unas jornadas de juegos de mesa, actividades de talleres callejeros dedicados a público general, y talleres de iniciación para público universitario, con una gran aceptación de participantes en todos los casos, y, aunque no disponemos de datos cuantitativos podemos expresar cualitativamente nuestras observaciones. Los ciudadanos de diversas edades se sentían muy a gusto jugando, perdían enseguida el temor a los juegos que no conocían y se tomaban muy en serio la tarea de cada juego, con una mezcla de competitividad y sentido del placer obtenido al jugar sin otra finalidad que el propio juego, como puede comprobarse en las imágenes contenidas en las figuras 1 a 4 .

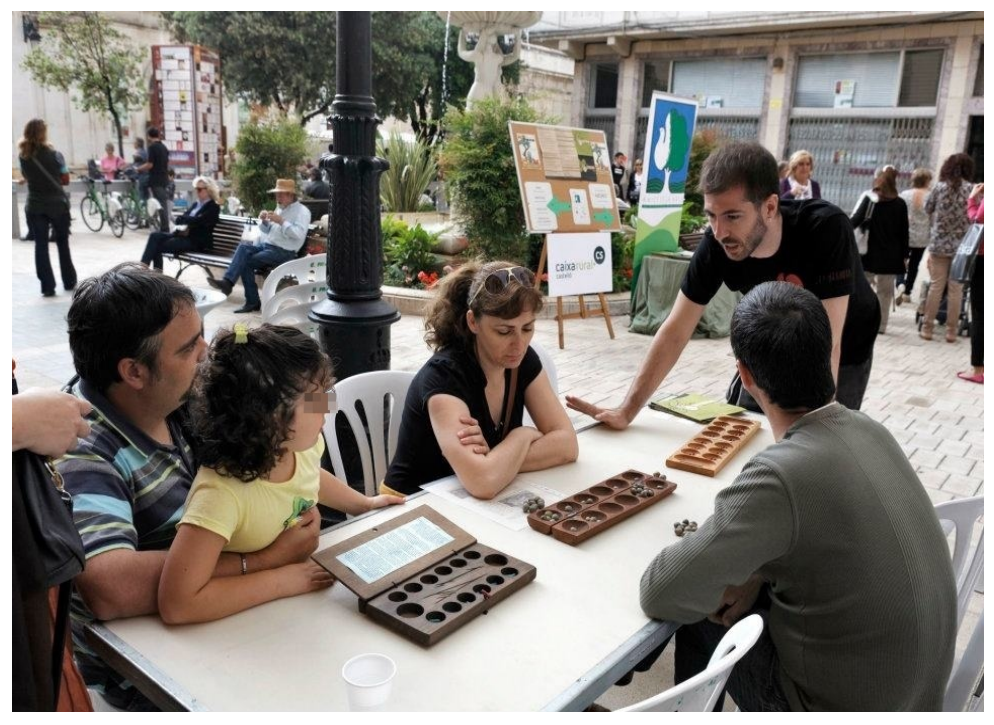

Figura 1: Explicando las reglas del juego a una familia de jugadores

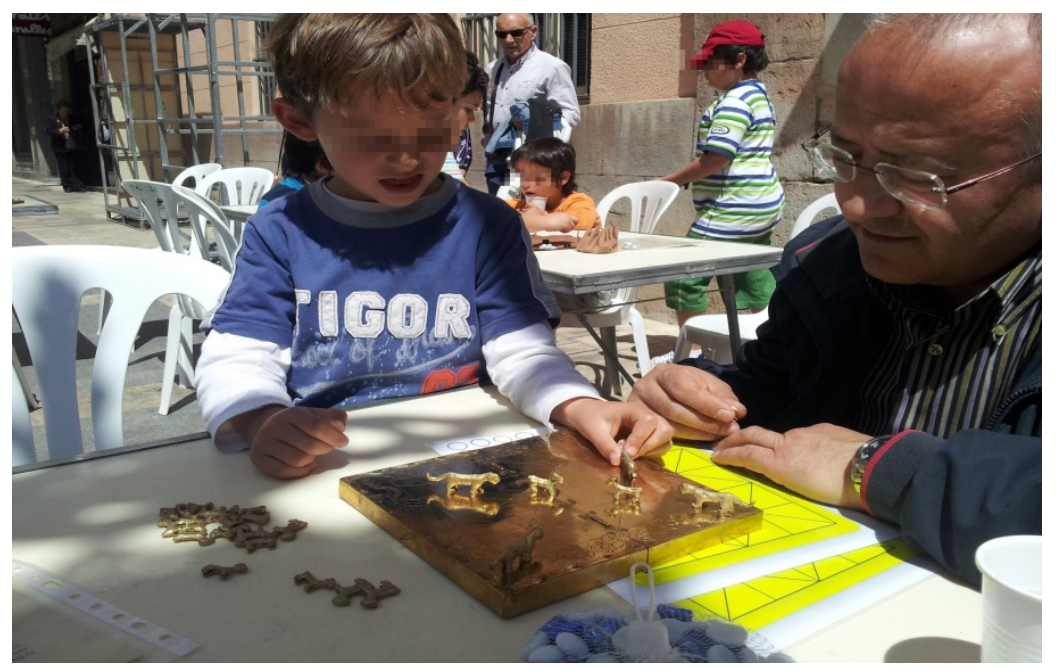

Figura 2: Niño muy interesado jugando con un adulto 


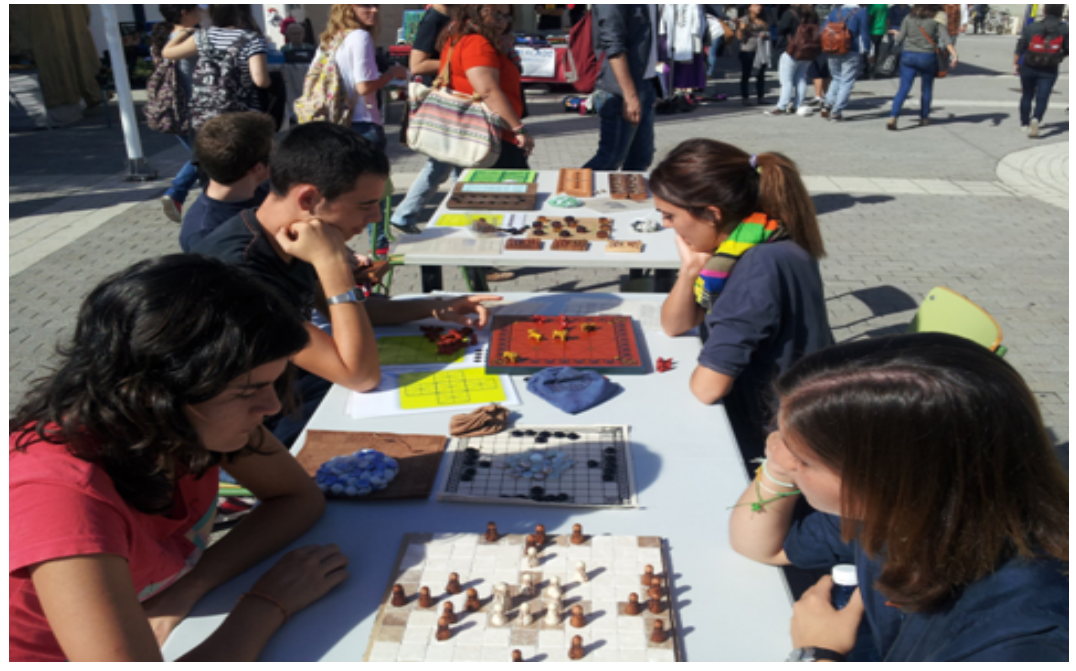

Figura 3: Jóvenes practicando diversos juegos ancestrales

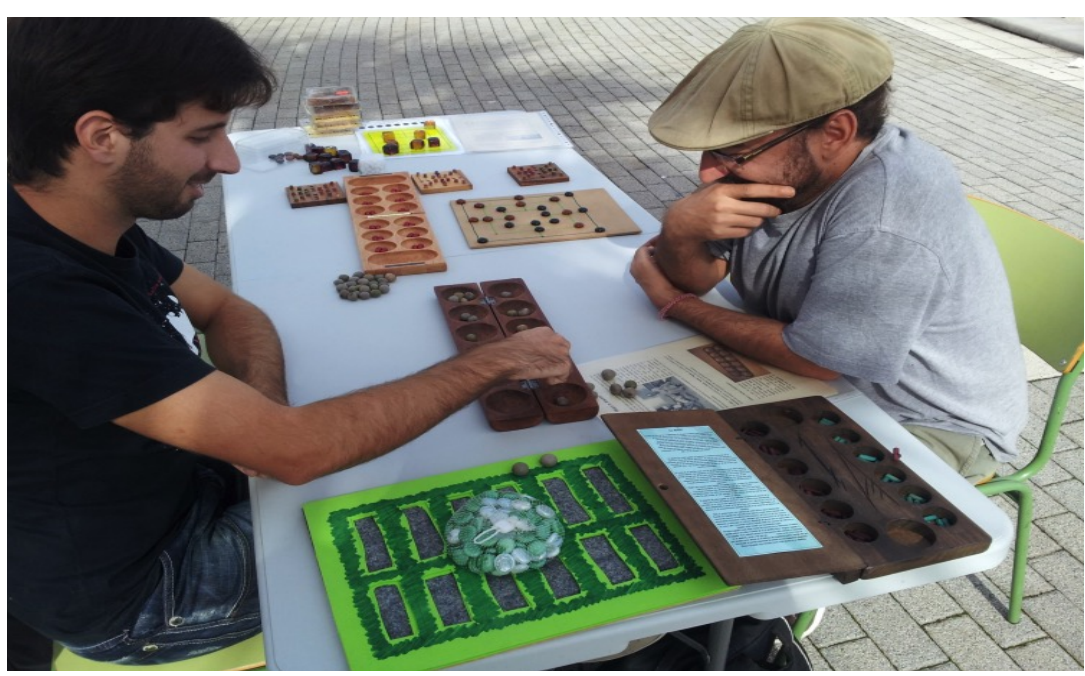

Figura 4: Estos juegos favorecen la capacidad de concentración

Por ello proponemos los siguientes juegos, cuidadosamente seleccionados a fin de cumplir con los objetivos previstos. Pueden ser clasificados mediante algunas características claves que los relacionan con contenidos de matemáticas y ciencias (materiales naturales: madera, semillas, piedras, metales, o sintéticos: papel, plástico, laca) bien en sus tableros y fichas de juego (Figura 5), bien en sus reglas (árboles dicotómicos, recuentos, azar, probabilidad, desplazamientos en el plano, composición de figuras geométricas), o en ambas componentes.

Se presentan en total 41 juegos agrupados en 8 categorías o familias designadas mediante una propiedad clave, citando en primer lugar los contenidos relativos a conceptos y procesos y a continuación los juegos correspondientes:

- Elementos de azar. Contenidos (2): azar, probabilidad como medida de incertidumbre. Elementos de azar: monedas y dados hexaedros, tablillas, cauris, maíz, tabas, dados. Juegos (7): Juegos de monedas: cara o cruz y lu-lu. Juegos de dedos: los chinos, pares o nones, piedra-papel-tijera, piedra-papel-tijera-lagarto- 
Spock. Juegos de tabas: ave victrix.

- Juegos de posición. Contenidos (5): planificación táctico-estratégica y pensamiento arborescente, detección de patrones regulares y análisis de simetrías. Juegos (8): Juegos de bloqueo: pong hau k'i y mu torere. Juegos de molino: tres en raya, danza de los seis, los nueve y los doce hombres. Otros: seega moderno y sz'kwa.

- Juegos de carreras. Contenidos (3): estrategia favorecedora, combinación táctica y azar. Juegos (4): Juego real de Ur y senet. Juegos de cruz y círculo: nyout y pachisi.

- Juegos de caza. Contenidos (4): planificación estratégica y pensamiento arborescente, detección de patrones regulares y análisis de simetrías. Juegos (4): Juegos de zorros: la zorra y los gansos. Juegos de leopardos: vacas y leopardos. Juegos de tigres: bagh chal y corderos y tigres.

- Juegos de guerra. Contenidos (4): planificación estratégica y pensamiento arborescente, detección de patrones regulares y análisis de simetrías. Juegos (10). Juegos de alquerque: alquerque, peralikatuma, fanorona y kolowis awithlaknannai. Juegos tafl: tablut y hnefatafl. Juegos de damas: damas del perdedor y halma. Otros: asalto y puluc.

- Juegos mancala. Contenidos (5): aritmética modular, agilidad de cálculo mental, planificación táctico-estratégica y pensamiento arborescente. Juegos (2): Juegos mancala II: kalaha y wari.

- Juegos modernos. Contenidos (5): planificación táctico-estratégica y pensamiento arborescente, detección de patrones regulares y análisis de simetrías. Juegos (2): Othello y Abalone.

- Solitarios. Contenidos (8): geometría, inducción y deducción, recursividad, pensamiento arborescente, sucesiones numéricas, detección de patrones, análisis de simetrías. Juegos (4): Tangram, las torres de Hanói, el solitario de la Bastilla o senku, y tchuka ruma.
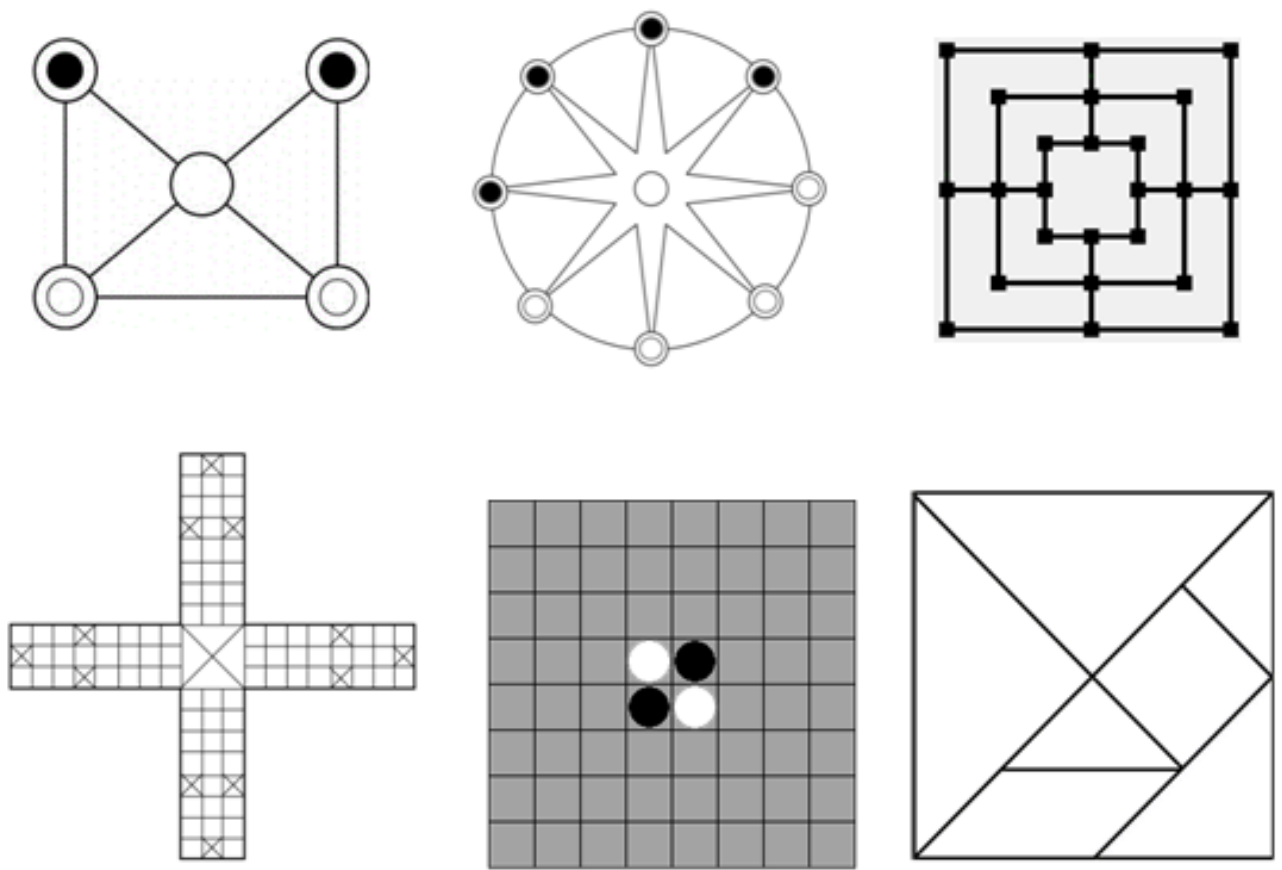

Figura 5: Tableros y posiciones iniciales de las fichas de algunos de los juegos seleccionados en la propuesta. De izquierda a derecha y de arriba abajo: pong hau k'i y mu torere (con un eje de simetría), danza de los nueve hombres y pachisi (con cuatro ejes de simetría), Othello (con dos ejes de simetría) y tangram (con cinco triángulos semejantes, un cuadrado, y un romboide. (Esquemas y comentarios de elaboración propia) 


\section{Conclusiones}

El potencial científico y matemático de estos 41 juegos es muy amplio, lo cual se evidencia en el análisis efectuado de ellos mediante el cual se han detectado inicialmente 8 categorías o familias de juegos que tienen elementos intrínsecos en común y 36 aspectos etnomatemáticos, es decir de matemáticas implícitas en las reglas, tableros y piezas del juego, que inducen al ejercicio mental mediante el cual se adquiere capacidad científica.

Una vez probados los juegos en actividades de talleres y jornadas lúdicas observamos que, aunque algunos presentan ciertas dificultades iniciales, esencialmente por desconocimiento, las ventajas son mucho más numerosas y significativas. Estos juegos, además de permitir conocer aspectos característicos de otras culturas, resultan muy útiles para la enseñanza-aprendizaje de las matemáticas y las ciencias, uno de los objetivos principales que se perseguía con la elaboración del catálogo presentado.

Jugar a estos juegos del mundo es motivador, puesto que libera el estrés de otras tareas mediante el reforzamiento de la faceta lúdica necesaria para el equilibrio psicológico. Por todo ello, el uso de los juegos ha resultado muy satisfactorio y enriquecedor en líneas generales.

\section{Referencias}

Albizu, U., Fernández-Oliveras, A., y Oliveras, M. L. (2014). Bases para investigar desde las Etnomatemáticas. En Sánchez, C. (Ed.), Líneas emergentes en la investigación de vanguardia, 27-40. Madrid: McGraw Hill.

Bishop, A. J. (1998). El papel de los juegos en educación matemática [en línea]. Uno. Revista de Didáctica de las Matemáticas, 18, 9-19. Recuperado en junio de 2015 de: http://dgespe.edutlixco.org/pdf/educa/pap_jueg.pdf

Decroly, O. y Monchamp, E. (1983). El juego educativo: iniciación a la actividad intelectual y motriz. Madrid: Ediciones Morata.

Elkonin, D. (1985). Psicología del juego. Madrid: Visor.

Fernández-Oliveras, A. y Oliveras, M. L. (2014a). Playing for science and mathematics education: an experience for pre-service kindergarten teacher training. En Costa M. F. M., Pombo P., Dorrío B.V. (Eds.), Hands-on Science. Science Education with and for Society (180 -183). Braga: Hands-on Science Network.

Fernández-Oliveras, A. y Oliveras, M. L. (2014b). Pre-service kindergarten teachers' conceptions of play, science, mathematics, and education. Procedia - Social and Behavioral Sciences, 152, 856-861. DOI:10.1016/j.sbspro.2014.09.334.

Fernández-Oliveras, A. y Oliveras, M. L. (2015). Conceptions of science, mathematics, and education of prospective kindergarten teachers in a play-based training. International Journal on Advances in Education Research, 2(1), 37-48.

Gómez-Lara, J. (2003). Los productos humanos, instrumentos de cambio para la educación intercultural [en línea]. Colectivo Amani, Aula Intercultural. Recuperado en junio de 2015 de: http://aulaintercultural.org/2003/12/08/los-productos-humanos-instrumentos-de-cambio-para-la-educacionintercultural/

Huizinga, J. (1972). Homo Ludens. Madrid: Alianza Editorial.

Oliveras, M.L. (2006). Etnomatemáticas. De la multiculturalidad al mestizaje. En Goñi, J. M. (Coord.), Matemáticas e interculturalidad, 117-149. Barcelona: Graó. Col. Biblioteca de Uno. 